\title{
Assessing foods offered in the Food Distribution Program on Indian Reservations (FDPIR) using the Healthy Eating Index 2010
}

\author{
Carmen Byker Shanks ${ }^{1, *}$, Teresa Smith ${ }^{2}$, Selena Ahmed ${ }^{1}$ and Holly Hunts ${ }^{3}$ \\ ${ }^{1}$ Food and Health Lab, Department of Health and Human Development, Montana State University, PO Box 173540, \\ Bozeman, MT 59717, USA: ${ }^{2}$ Department of Health Promotion, Social and Behavioral Health, University of Nebraska \\ Medical Center, Omaha, NE, USA: ${ }^{3} 222$ Herrick Hall, Montana State University, Bozeman, MT, USA
}

Submitted 12 September 2014: Final revision received 14 June 2015: Accepted 6 July 2015: First published online 24 September 2015

\begin{abstract}
Objective: To assess the nutritional quality of food packages offered in the Food Distribution Program on Indian Reservations (FDPIR) using the Healthy Eating Index 2010 (HEI-2010).

Design: Data were collected from the list of the food products provided by the US Department of Agriculture's Food and Nutrition Handbook 501 for FDPIR. Nutritional quality was measured through a cross-sectional analysis of five randomly selected food packages offered through FDPIR. HEI-2010 component and total scores were calculated for each food package. ANOVA and $t$ tests assessed significant differences between food packages and HEI-2010 maximum scores, respectively.

Setting: This study took place in the USA.

Subjects: Study units included food products offered through FDPIR.

Results: The mean total HEI-2010 score for the combined FDPIR food packages was significantly lower than the total HEI-2010 maximum score of $100(66.38$ (SD 11.60); $P<0.01)$. Mean scores for total fruit $(3.52$ (SD 0.73 ); $P<0.05$ ), total vegetables (2.58 (sD 0.15); $P<0.001)$, greens and beans (0.92 (sD 1.00$) ; P<0.001)$, dairy (5.12 (SD 0.63); $P<0.001)$, total protein foods (4.14 (SD 0.56); $P<0.05)$ and refined grains $(3.04$ (SD 2.90); $P<0.001)$ were all significantly lower than the maximum values.

Conclusions: The FDPIR food package HEI-2010 score was notably higher than other federal food assistance and nutrition programmes. Study findings highlight opportunities for the FDPIR to modify its offerings to best support lifestyles towards prevention of diet-related chronic disease.
\end{abstract}

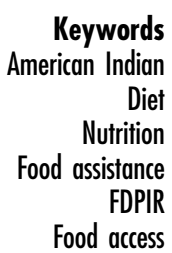

Overweight, obesity and nutrition-related chronic diseases are complex health conditions influenced by a number of biological, behavioural, environmental, genetic and personal factors ${ }^{(1)}$. Improving access to nutrient-dense foods is one key strategy to prevent nutrition-related chronic disease and obesity ${ }^{(2,3)}$. In the USA, access to nutrientdense foods is particularly a concern in communities with marked health disparities, including those that are rural, urban, of limited income or have high a percentage of minorities $^{(4-9)}$.

For example, American Indians are more likely than the general US population to live in rural locations with limited food access ${ }^{(10)}$. At the same time, American Indian adults are $60 \%$ more likely to be obese than non-Hispanic whites $^{(11)}$. The consequences of obesity are well documented, including the risk of developing diabetes mellitus ${ }^{(12)}$, which is particularly concerning as American
Indian and Alaska Natives have a higher age-adjusted prevalence of diabetes mellitus than any other race or ethnic group in the $\mathrm{USA}^{(13)}$.

Previous research ${ }^{(14,15)}$ indicates that the modern American Indian diet is poor in nutrient quality and household food security is relatively low ${ }^{(15-17)}$. Emerging research indicates potential connections between diets poor in nutrient quality, high food insecurity rates and high obesity and chronic disease rates among American Indians ${ }^{(18,19)}$. Contemporary food issues observed within Native American populations have been connected to a long and storied history of colonialism and historical trauma ${ }^{(20-22)}$. With socio-economic, political and environmental changes including reduction in tribal land, end of nomadic lifestyles, shifts in farming policies, the near extinction of buffalo and limited rights to hunt, fish and collect wild foods, the current diet among American 
Indians has transitioned notably from traditional ways in post-colonial times ${ }^{(23,24)}$.

In effort to address nutrition-related challenges faced by American Indian peoples, the US government has supplied food to American Indians living on reservations for over 150 years as well as had a series of food-related agreements ${ }^{(14)}$. For example, some treaties included 'annuities' which granted hunting, fishing and gathering rights for American Indians ${ }^{(25)}$. During the period circa 1860-1934, the government issued rations to supplement lost sources of wild foods and failed crops ${ }^{(26)}$. However, some historical documents describe the rations provided by the government as being culturally inappropriate, inadequate, not delivered as promised and of low quality ${ }^{(27,28)}$.

The Food Distribution Program on Indian Reservations (FDPIR) was implemented by Congress in 1973 as part of the Consumer Protection Act ${ }^{(29)}$. The Program states, 'many households participate in FDPIR as an alternative to the Supplemental Nutrition Assistance Program (SNAP), because they do not have easy access to SNAP offices or authorized food stores'(30).

Through FDPIR, the US Department of Agriculture (USDA) provides increased access to nutritious foods for low-income households living on Indian reservations and to American Indian families residing in designated areas near reservations ${ }^{(30)}$. The FDPIR is one of sixteen distinct federal food assistance and nutrition programmes administered by the Food and Nutrition Service of the $\mathrm{USDA}^{(31)}$. The programme provides individuals an alternative to the Supplemental Nutrition Assistance Program (SNAP) enrolment by directly distributing commodity packages in communities and striving to meet basic nutrient needs of programme participants ${ }^{(30)}$.

The USDA administers the FDPIR through either Indian Tribal Organizations (ITO) or an agency of a state government ${ }^{(32)}$. The USDA purchases and ships FDPIR foods to the ITO and state agencies based on orders placed from a list of available foods ${ }^{(32)}$. State agencies and ITO are responsible for determining applicant eligibility, storing and distributing the food, and providing nutrition education to recipients. According to the Program, 'Low-income American Indian and non-Indian households that reside on a reservation and households living in approved areas near a reservation or in Oklahoma that contain at least one person who is a member of a federally-recognized tribe, are eligible to participate in FDPIR $^{(30)}$. Households may not participate in the FDPIR and SNAP in the same month ${ }^{(32)}$. There currently are 276 tribes through 100 ITO and five state agencies receiving FDPIR benefits ${ }^{(30)}$. Since the inception of the FDPIR, participant size has increased with a total 75608 participants in $2013^{(33)}$. Each month, participants select a food package based on their food preferences, household size and foods available at their particular ITO or state agency distribution site to help them maintain a nutritionally balanced diet ${ }^{(32)}$.

In 2008, the Special Nutrition Programs Report no. FD-08-FDPIR was developed by the USDA to assess the nutritional quality of FDPIR foods utilizing the Healthy Eating Index 2005 (HEI-2005) $^{(34)}$. Results from the report indicated that FDPIR had the potential to provide participants with a diet of higher nutrient quality than the average American or SNAP participant.

To the best of the authors' knowledge, the Healthy Eating Index 2010 (HEI-2010) ${ }^{(35)}$ has yet to be utilized in assessing the nutritional quality of foods offered as part of FDPIR. The HEI-2010 ${ }^{(35)}$ has been developed to measure adherence to the most recently published federal dietary guidelines, the 2010 Dietary Guidelines for Americans $(\text { DGA })^{(36)}$, whereas HEI-2005 was developed to measure the previous version of the federal dietary guidelines, the 2005 Dietary Guidelines for Americans (2005 DGA) ${ }^{(35,37)}$. Specifically, HEI-2010 updates include: (i) emphasis on Dark Green Vegetables and Beans and Peas; (ii) a Seafood and Plant Proteins component was introduced; (iii) Fatty Acids replaces the Oils and Saturated Fats components; and (iv) Refined Grains (a moderation component) replaced Total Grains (an adequacy component) ${ }^{(35)}$.

It is important to assess the nutritional quality of FDPIR foods utilizing the HEI-2010 to understand how each iteration of current dietary guidance is reflected within the offerings of the food assistance programme. For example, dark green vegetables and beans and peas are two vegetable subgroups for which intakes are furthest from recommended levels and the category of 'vegetables and soup' allows for choices among many vegetables; the introduction of seafood and plant proteins within HEI-2010 allows for capturing the dietary contribution of more specific protein choices within the broad 'meat, poultry, fish, beans, eggs and nuts' category of FDPIR; replacing saturated fats with fatty acids within HEI-2010 allows for the more specific assessment of the value of vegetable oil, light buttery spread and butter within the 'oil' category of FDPIR; refined and whole grains are both offered within the FDPIR 'grains, cereal, rice and pasta' category and assessing these separately with HEI-2010 is important to understand their distinct dietary contributions ${ }^{(38,39)}$.

The sum of the scores for the twelve components is the total HEI-2010 score, which ranges from 0 to 100, with a higher score indicative of a more healthful diet. HEI-2010 is composed of twelve components, nine that focus on nutritional adequacy and three that apply nutritional moderation $^{(40)}$. For HEI-2010, Refined Grains, Sodium and Empty Calories are all moderation components. A higher score within moderation components indicates lower availability of the food in the diet. All other categories are adequacy components, where a higher score indicates higher availability of food in the diet. HEI-2010 scores separate diet quality from quantity by using standards that are expressed as a percentage of energy, per $1000 \mathrm{kcal}$ $(4184 \mathrm{~kJ})$ or a ratio of fatty acids ${ }^{(40)}$.

The lack of assessment of the FDPIR with the HEI-2010 presents a knowledge gap regarding the dietary quality of FDPIR foods that support American Indian households in 
compliance with the 2010 DGA. Current nutrition research is needed in order to develop appropriate nutritional planning and policies related to food assistance, food security and obesity in tribal communities with marked health disparities. The purpose of the current research was to assess the nutritional quality of foods offered in the FDPIR using HEI-2010.

\section{Experimental methods}

Data were collected from a list of the food products found in Exhibit $\mathrm{O}$ of the Food and Nutrition Handbook 501 for FDPIR, which was effective as of September $2013^{(32)}$. The study was exempt from Institutional Review Board review since no information was collected from human subjects.

\section{Data analysis}

Each food option was entered into the USDA What's In The Foods You Eat online search tool (version 5.0) ${ }^{(41)}$. Matching food package components and search tool foods was based on the item description and nutrient profiles. Each food item was assigned a USDA food code and nutrient composition was ascertained (Table 1). Food group composition was determined using the MyPyramid Equivalents Database for USDA Survey Food Codes, 20032004 (version 2). Each food listed in FDPIR, including foods requiring preparation (e.g. flour) and the few available ready-to-eat options, can be found in the cited database ${ }^{(41)}$.

The researchers simulated five possible food package scenarios for analysis by (i) using the FDPIR guide to establish the maximum allowed number of items for a one-person household ${ }^{(39)}$ and then (ii) randomly selecting the maximum allowed number of items per USDA food group ('grains, cereal, rice and pasta'; 'vegetables and soup'; 'fruit and juice'; 'meat, poultry, fish, beans, eggs and nuts'; 'milk and cheese'; 'oil'). The FDPIR guide outlines requirements for the number of items that can be chosen based on the number of people in a household per month for each food item ${ }^{(39)}$. The number of items that can be chosen is often increased linearly per person (e.g. 1 person $=1$ item, 2 persons $=2$ items, 3 persons $=3$ items, etc.). Analysis was based on a one-person household with the expectation that the dietary quality would remain consistent with increasing number of persons in a household. For each food package, a random number generator was utilized to randomly select from all options per USDA food group. Randomly generated options were allowed to be chosen more than once when FDPIR guidelines allowed for greater than one option per USDA food group.

Using randomly generated food packages, HEI-2010 component and total scores were calculated using published SAS code (version 9.2), modified to assess this specific data set ${ }^{(42)}$. Prior to analysis, ANOVA was used to detect if the criteria for randomly selecting food packages used in the present study could lead to significant differences in key nutrient content across each of the five food packages. No significant differences were found among total energy, carbohydrates $(\mathrm{g})$, saturated fat $(\mathrm{g})$ and $\mathrm{Na}$ (mg) for each of the five food packages.

Following the methodology outlined by Erinosho and colleagues $^{(43)}$, means and standard deviations were calculated to generate both HEI-2010 component scores and total scores across all menus. The $t$ test was calculated to assess whether mean HEI-2010 component scores and total scores differed significantly $(P<0.05)$ from the maximum scores.

\section{Results}

Table 2 describes HEI-2010 component scores and total scores for foods and beverages provided as part of the five randomly generated FDPIR food packages. The mean total HEI-2010 score for the combined FDPIR food packages was significantly lower than the total HEI-2010 maximum score of $100(66.38$ (SD 11.60); $P<0.01)$, with total HEI-2010 scores ranging from $49 \cdot 50$ to 79.50 across all five FDPIR food packages. Mean scores for Total Fruit (3.52 (SD 0.73); $P<0.05$ ), Total Vegetables (2.58 (SD 0.15); $P<0.001)$, Greens and Beans (0.92 (SD 1.00); $P<0.001)$, Dairy (5.12 (sD 0.63); $P<0.001$ ), Total Protein Foods (4.14 (SD 0.56); $P<0.05$ ) and Refined Grains (3.04 (SD 2.90); $P<0 \cdot 01)$ were all significantly lower than the maximum values (of 5, 5, 5, 10, 5 and 10, respectively). All other components did not demonstrate significant differences from their maximum values.

Contributing to the combined FDPIR HEI-2010 score, all five food packages (100\%) met the standard for a maximum value for Empty Calories, followed by three (60\%) that met the standard for Whole Grains, three (60\%) that met the standard for Seafood and Plant Proteins, two ( $40 \%$ ) that met the standard for Whole Fruit and one (20\%) that met the standard for Fatty Acids. No sample food packages met the standard for a maximum value for Total Fruit, Total Vegetables, Greens and Beans, Dairy, Total Protein Foods, Refined Grains or Sodium.

\section{Discussion}

The present study addresses an important knowledge gap by characterizing the mean nutritional quality of five randomly generated food packages of the FDPIR on the basis of the most recently published federal dietary guidelines, the 2010 DGA. The FDPIR packages are not meeting the diet quality recommendations outlined by the 2010 DGA, as our analysis found significantly lower HEI-2010 overall score compared with the maximum score.

Similar to our findings, Americans do not consume adequate amounts of fruits, vegetables, whole grains or dairy and significantly lower HEI-2010 component scores compared with the maximum values from 2010 DGA were 
Table 1 USDA food codes and foods for five sample monthly FDPIR food packages

\begin{tabular}{|c|c|c|}
\hline $\begin{array}{l}\text { Food package, food group and } \\
\text { USDA food code }\end{array}$ & Grams per food item & Food item \\
\hline \multicolumn{3}{|l|}{ Food Package 1} \\
\hline \multicolumn{3}{|l|}{ Grains, cereal, rice and pasta } \\
\hline 57134000 & 400 & Corn flakes, NFS \\
\hline 56206990 & 2744 & $\begin{array}{l}\text { Wheat, cream of, cooked, NS as to regular, quick or instant, NS as to fat added in } \\
\text { cooking }\end{array}$ \\
\hline 56101000 & 1248 & Macaroni, cooked, NS as to fat added in cooking $(\times 2)$ \\
\hline 56112000 & 1184 & Noodles, cooked, NS as to fat added in cooking \\
\hline 56205330 & 2880 & Rice, white and wild, cooked, NS as to fat added in cooking \\
\hline 50020000 & 2250 & Flour, whole wheat $(\times 2)$ \\
\hline 50010000 & 2250 & Flour, white $(\times 0.25)$ \\
\hline 54325000 & 453 & Crackers, saltine \\
\hline \multicolumn{3}{|l|}{ Vegetables and soup } \\
\hline 73102203 & 440 & Carrots, cooked, from canned, NS as to fat added in cooking \\
\hline 75216050 & 440 & Corn, NS as to form, NS as to colour, cream style \\
\hline 73201003 & 440 & Pumpkin, cooked, from canned, NS as to fat added in cooking \\
\hline 73101010 & 488 & Carrots, raw \\
\hline 75117020 & 440 & Onions, mature, raw \\
\hline 73401000 & 238 & Sweet potato, NFS \\
\hline 75103000 & 908 & Cabbage, green, raw \\
\hline 75125000 & 416 & Radish, raw \\
\hline 75109600 & 429 & Corn, raw \\
\hline 74101000 & $300 \cdot 2$ & Tomatoes, raw \\
\hline 75122100 & 357 & Pepper, sweet, green, raw \\
\hline 28315100 & 720 & Beef vegetable soup with potato, stew type $(\times 2)$ \\
\hline 74601000 & $320 \cdot 2$ & Tomato soup, NFS \\
\hline \multicolumn{3}{|l|}{ Fruit and juice } \\
\hline 63101000 & 546 & Apple, raw $(\times 2)$ \\
\hline 61101010 & 512 & Grapefruit, raw $(\times 2)$ \\
\hline 63137010 & 534 & Pear, raw \\
\hline 63127010 & 640 & Honeydew melon, raw \\
\hline 63126500 & 414 & Kiwi fruit, raw \\
\hline 63143010 & 198 & Plum, raw \\
\hline 62122100 & 387.5 & Prune, dried, uncooked \\
\hline 64104010 & 1984 & Apple juice \\
\hline 61201220 & $1977 \cdot 6$ & Grapefruit juice, canned, bottled or in a carton \\
\hline \multicolumn{3}{|c|}{ Meat, poultry, fish, beans, eggs and nuts } \\
\hline 21500000 & 453.6 & Ground beef, raw \\
\hline 23326100 & 352 & Bison, cooked \\
\hline 21401000 & 704 & Beef, roast, roasted, NS as to fat eaten \\
\hline 22311000 & 368 & Ham, smoked or cured, cooked, NS as to fat eaten \\
\hline 41106000 & 279 & Red kidney beans, dry, cooked, NS as to fat added in cooking \\
\hline 41205010 & $447 \cdot 6$ & Refried beans $(\times 2)$ \\
\hline 41104000 & 310 & Pinto, calico or red Mexican beans, dry, cooked, NS as to fat added in cooking \\
\hline 33102010 & 360 & Scrambled egg, made from powdered mixture $(\times 2)$ \\
\hline 42501000 & 420 & Nut mixture with dried fruit and seeds \\
\hline \multicolumn{3}{|l|}{ Milk and cheese } \\
\hline 14410200 & 2268 & Cheese, processed, American or Cheddar type $(\times 0.5)$ \\
\hline 11212050 & 384 & Milk, evaporated, skimmed (formerly NS as to dilution, used in coffee or tea) $(\times 4)$ \\
\hline 11112210 & 976 & Milk, cow's, fluid, $1 \%$ fat $(\times 4)$ \\
\hline \multicolumn{3}{|r|}{ 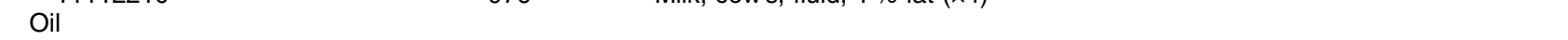 } \\
\hline 82101000 & 1308 & Vegetable oil, NFS \\
\hline \multicolumn{3}{|l|}{ Food Package 2} \\
\hline \multicolumn{3}{|l|}{ Grains, cereal, rice and pasta } \\
\hline 57207000 & 400 & Bran flakes, NFS (formerly $40 \%$ bran flakes, NFS) \\
\hline 57602100 & 1200 & Oats, raw \\
\hline 58145110 & 200 & Macaroni or noodles with cheese $(\times 3)$ \\
\hline 56101000 & 1248 & Macaroni, cooked, NS as to fat added in cooking \\
\hline 56102000 & 1248 & Macaroni, whole wheat, cooked, NS as to fat added in cooking \\
\hline 56205330 & 2880 & Rice, white and wild, cooked, NS as to fat added in cooking \\
\hline 56201510 & 14640 & Cornmeal mush, made with water \\
\hline 50020000 & 2250 & Flour, whole wheat \\
\hline 50010000 & 2250 & Flour, white $(\times 0.25)$ \\
\hline 54325000 & 453 & Crackers, saltine \\
\hline \multicolumn{3}{|l|}{ Vegetables and soup } \\
\hline 56200990 & 440 & $\begin{array}{l}\text { Grits, cooked, corn or hominy, NS as to regular, quick or instant, NS as to fat } \\
\text { added in cooking }\end{array}$ \\
\hline 74404010 & 440 & Spaghetti sauce, meatless \\
\hline 73101010 & 488 & Carrots, raw \\
\hline 71000100 & 334 & White potato, NFS \\
\hline
\end{tabular}


Table 1 Continued

\begin{tabular}{|c|c|c|}
\hline $\begin{array}{l}\text { Food package, food group and } \\
\text { USDA food code }\end{array}$ & Grams per food item & Food item \\
\hline 73302010 & 280 & Squash, winter type, raw \\
\hline 75128000 & 392 & Squash, summer, yellow, raw \\
\hline 73401000 & 238 & Sweet potato, NFS \\
\hline 75103000 & 908 & Cabbage, green, raw $(\times 2)$ \\
\hline 75109000 & 400 & Celery, raw \\
\hline 75111000 & 402 & Cucumber, raw \\
\hline 75607030 & 305 & Mushroom soup, canned, undiluted $(\times 3)$ \\
\hline \multicolumn{3}{|l|}{ Fruit and juice } \\
\hline 63311110 & $437 \cdot 9$ & $\begin{array}{l}\text { Fruit cocktail, cooked or canned, NS as to sweetened or unsweetened; } \\
\text { sweetened, NS as to type of sweetener }(\times 2)\end{array}$ \\
\hline 63105010 & 402 & Avocado, raw \\
\hline 63311050 & 440 & Fruit salad, fresh or raw, (including citrus fruits), no dressing \\
\hline 63123000 & 377.5 & Grapes, raw, NS as to type $(\times 3)$ \\
\hline 63126500 & 414 & Kiwi fruit, raw \\
\hline 63143010 & 198 & Plum, raw \\
\hline 62122100 & 387.5 & Prune, dried, uncooked \\
\hline 64116020 & $1996 \cdot 8$ & Grape juice \\
\hline 61210000 & 1990.4 & Orange juice, NFS \\
\hline \multicolumn{3}{|c|}{ Meat, poultry, fish, beans, eggs and nuts } \\
\hline 24198570 & 600 & Chicken, canned, meat only \\
\hline 23326100 & 352 & Bison, cooked \\
\hline 24201310 & 960 & Turkey, light and dark meat, roasted, NS as to skin eaten \\
\hline 22311000 & 368 & Ham, smoked or cured, cooked, NS as to fat eaten \\
\hline 41101100 & 2240 & White beans, dry, cooked, NS as to fat added in cooking \\
\hline 33102010 & 360 & Scrambled egg, made from powdered mixture $(\times 2)$ \\
\hline 42501000 & 420 & Nut mixture with dried fruit and seeds \\
\hline \multicolumn{3}{|l|}{ Milk and cheese } \\
\hline 14410200 & 2268 & Cheese, processed, American or Cheddar type $(\times 0.5)$ \\
\hline 11212050 & 384 & Milk, evaporated, skimmed (formerly NS as to dilution, used in coffee or tea) $(\times 4)$ \\
\hline 11121300 & $2587 \cdot 2$ & Milk, dry, reconstituted, non-fat $(\times 0.5)$ \\
\hline \multicolumn{3}{|r|}{ 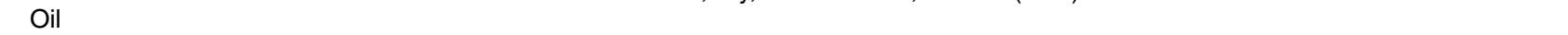 } \\
\hline 82101000 & 1308 & Vegetable oil, NFS \\
\hline \multicolumn{3}{|l|}{ Food Package 3} \\
\hline \multicolumn{3}{|l|}{ Grains, cereal, rice and pasta } \\
\hline 57000100 & 400 & Oat cereal, NFS \\
\hline 56206990 & 2744 & $\begin{array}{l}\text { Wheat, cream of, cooked, NS as to regular, quick or instant, NS as to fat added in } \\
\text { cooking }\end{array}$ \\
\hline 58145110 & 200 & Macaroni or noodles with cheese $(\times 3)$ \\
\hline 56112000 & 1184 & Noodles, cooked, NS as to fat added in cooking $(\times 2)$ \\
\hline 56205330 & 2880 & Rice, white and wild, cooked, NS as to fat added in cooking \\
\hline 56201510 & 14640 & Cornmeal mush, made with water \\
\hline 50010000 & 2250 & Flour, white \\
\hline 50010000 & 2250 & Flour, white $(\times 0.25)$ \\
\hline 54325000 & 453 & Crackers, saltine \\
\hline \multicolumn{3}{|l|}{ Vegetables and soup } \\
\hline 75216050 & 440 & Corn, NS as to form, NS as to colour, cream style \\
\hline 71501300 & 440 & White potato, from dry, mashed, NS as to milk or fat $(\times 2)$ \\
\hline 74404010 & 440 & Spaghetti sauce, meatless \\
\hline 73201003 & 440 & Pumpkin, cooked, from canned, NS as to fat added in cooking \\
\hline 73101010 & 400 & Carrots, raw \\
\hline 75129000 & 366 & Turnip, raw \\
\hline 75103000 & 908 & Cabbage, green, raw \\
\hline 75102750 & 416 & Brussels sprouts, raw \\
\hline 72116000 & 376 & Endive, chicory, escarole or romaine lettuce, raw \\
\hline 74101000 & $300 \cdot 2$ & Tomatoes, raw \\
\hline 28315100 & 720 & Beef vegetable soup with potato, stew type \\
\hline 74601000 & $320 \cdot 2$ & Tomato soup, NFS $(\times 2)$ \\
\hline \multicolumn{3}{|l|}{ Fruit and juice } \\
\hline 63103110 & 425 & $\begin{array}{l}\text { Apricot, cooked or canned, NS as to sweetened or unsweetened; sweetened, NS } \\
\text { as to type of sweetener }\end{array}$ \\
\hline 63137110 & $437 \cdot 9$ & $\begin{array}{l}\text { Pear, cooked or canned, NS as to sweetened or unsweetened; sweetened, NS as } \\
\text { to type of sweetener }\end{array}$ \\
\hline 63105010 & 402 & Avocado, raw \\
\hline 61119010 & 393 & Orange, raw \\
\hline 63135010 & 450 & Peach, raw \\
\hline 63123000 & 377.5 & Grapes, raw, NS as to type $(\times 2)$ \\
\hline 63127010 & 640 & Honeydew melon, raw \\
\hline 63131010 & 408 & Nectarine, raw \\
\hline
\end{tabular}


Table 1 Continued

\begin{tabular}{|c|c|c|}
\hline $\begin{array}{l}\text { Food package, food group and } \\
\text { USDA food code }\end{array}$ & Grams per food item & Food item \\
\hline 62125100 & $439 \cdot 4$ & Raisins \\
\hline 64116020 & $1996 \cdot 8$ & Grape juice \\
\hline 61210000 & $1990 \cdot 4$ & Orange juice, NFS \\
\hline \multicolumn{3}{|c|}{ Meat, poultry, fish, beans, eggs and nuts } \\
\hline 21500000 & $453 \cdot 6$ & Ground beef, raw \\
\hline 23326100 & 352 & Bison, cooked \\
\hline 24100000 & 1152 & Chicken, NS as to part and cooking method, NS as to skin eaten \\
\hline 22311000 & 368 & Ham, smoked or cured, cooked, NS as to fat eaten \\
\hline 41205010 & 447.6 & Refried beans \\
\hline 41102000 & 342.9 & Black, brown or bayo beans, dry, cooked, NS as to fat added in cooking \\
\hline 41104000 & 310 & $\begin{array}{l}\text { Pinto, calico or red Mexican beans, dry, cooked, NS as to fat added in } \\
\text { cooking }(\times 2)\end{array}$ \\
\hline 33102010 & 360 & Scrambled egg, made from powdered mixture $(\times 2)$ \\
\hline 42202000 & 256 & Peanut butter \\
\hline \multicolumn{3}{|l|}{ Milk and cheese } \\
\hline 14410200 & 2268 & Cheese, processed, American or Cheddar type $(\times 0.5)$ \\
\hline 11212050 & 384 & Milk, evaporated, skimmed (formerly NS as to dilution, used in coffee or tea) $(\times 4)$ \\
\hline 11121300 & $2587 \cdot 2$ & Milk, dry, reconstituted, non-fat $(\times 0.5)$ \\
\hline \multicolumn{3}{|r|}{ 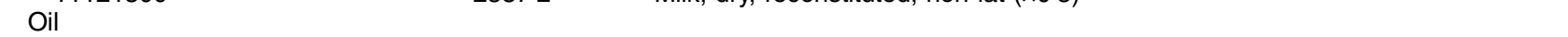 } \\
\hline 81104010 & 425 & Margarine-like spread, reduced calorie, about $40 \%$ fat, tub, salted $(\times 2)$ \\
\hline \multicolumn{3}{|r|}{ 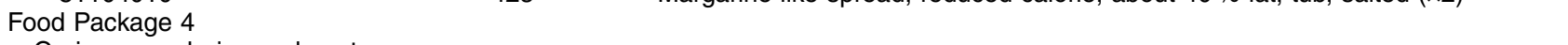 } \\
\hline \multicolumn{3}{|l|}{ Grains, cereal, rice and pasta } \\
\hline 57207000 & 400 & Bran flakes, NFS (formerly $40 \%$ bran flakes, NFS) \\
\hline 57602100 & 1200 & Oats, raw \\
\hline 56101000 & 1248 & Macaroni, cooked, NS as to fat added in cooking \\
\hline 56102000 & 1248 & Macaroni, whole wheat, cooked, NS as to fat added in cooking $(\times 2)$ \\
\hline 56205330 & 2880 & Rice, white and wild, cooked, NS as to fat added in cooking \\
\hline 56201510 & 14640 & Cornmeal mush, made with water \\
\hline 50010000 & 2250 & Flour, white \\
\hline 50010000 & 2250 & Flour, white $(\times 0.25)$ \\
\hline 54325000 & 453 & Crackers, saltine \\
\hline \multicolumn{3}{|l|}{ Vegetables and soup } \\
\hline 56200990 & 440 & $\begin{array}{l}\text { Grits, cooked, corn or hominy, NS as to regular, quick or instant, NS as to fat } \\
\text { added in cooking }\end{array}$ \\
\hline 75224013 & 440 & Peas, green, cooked, from canned, NS as to fat added in cooking \\
\hline 72125203 & 440 & Spinach, cooked, from canned, NS as to fat added in cooking \\
\hline 71501300 & 440 & White potato, from dry, mashed, NS as to milk or fat \\
\hline 73101010 & 400 & Carrots, raw $(\times 2)$ \\
\hline 71000100 & 334 & White potato, NFS \\
\hline 75102750 & 416 & Brussels sprouts, raw \\
\hline 72116000 & 376 & Endive, chicory, escarole or romaine lettuce, raw \\
\hline 74101000 & 298 & Tomatoes, raw \\
\hline 74101000 & $300 \cdot 2$ & Tomatoes, raw \\
\hline 28315100 & 720 & Beef vegetable soup with potato, stew type $(\times 2)$ \\
\hline 74601000 & 320.2 & Tomato soup, NFS \\
\hline \multicolumn{3}{|l|}{ Fruit and juice } \\
\hline 63101110 & $437 \cdot 9$ & $\begin{array}{l}\text { Applesauce, stewed apples, NS as to sweetened or unsweetened; sweetened, } \\
\text { NS as to type of sweetener }(\times 2)\end{array}$ \\
\hline 63103110 & 425 & $\begin{array}{l}\text { Apricot, cooked or canned, NS as to sweetened or unsweetened; sweetened, NS } \\
\text { as to type of sweetener }(\times 2)\end{array}$ \\
\hline 63311110 & $437 \cdot 9$ & $\begin{array}{l}\text { Fruit cocktail, cooked or canned, NS as to sweetened or unsweetened; } \\
\text { sweetened, NS as to type of sweetener }\end{array}$ \\
\hline 61101010 & 512 & Grapefruit, raw \\
\hline 63137010 & 534 & Pear, raw \\
\hline 63135010 & 450 & Peach, raw \\
\hline 63127010 & 640 & Honeydew melon, raw \\
\hline 63131010 & 408 & Nectarine, raw \\
\hline 64116020 & $1996 \cdot 8$ & Grape juice \\
\hline 74301100 & $1945 \cdot 6$ & Tomato juice \\
\hline \multicolumn{3}{|c|}{ Meat, poultry, fish, beans, eggs and nuts } \\
\hline 22101000 & 336 & Pork chop, NS as to cooking method, NS as to fat eaten $(\times 2)$ \\
\hline 21401000 & 704 & Beef, roast, roasted, NS as to fat eaten \\
\hline 22311000 & 368 & Ham, smoked or cured, cooked, NS as to fat eaten \\
\hline 41201020 & $492 \cdot 1$ & Baked beans, vegetarian \\
\hline 41205010 & 447.6 & Refried beans \\
\hline 41104000 & 310 & $\begin{array}{l}\text { Pinto, calico or red Mexican beans, dry, cooked, NS as to fat added in } \\
\text { cooking }(\times 2)\end{array}$ \\
\hline 33102010 & 360 & Scrambled egg, made from powdered mixture $(\times 2)$ \\
\hline 42202000 & 256 & Peanut butter \\
\hline
\end{tabular}


Table 1 Continued

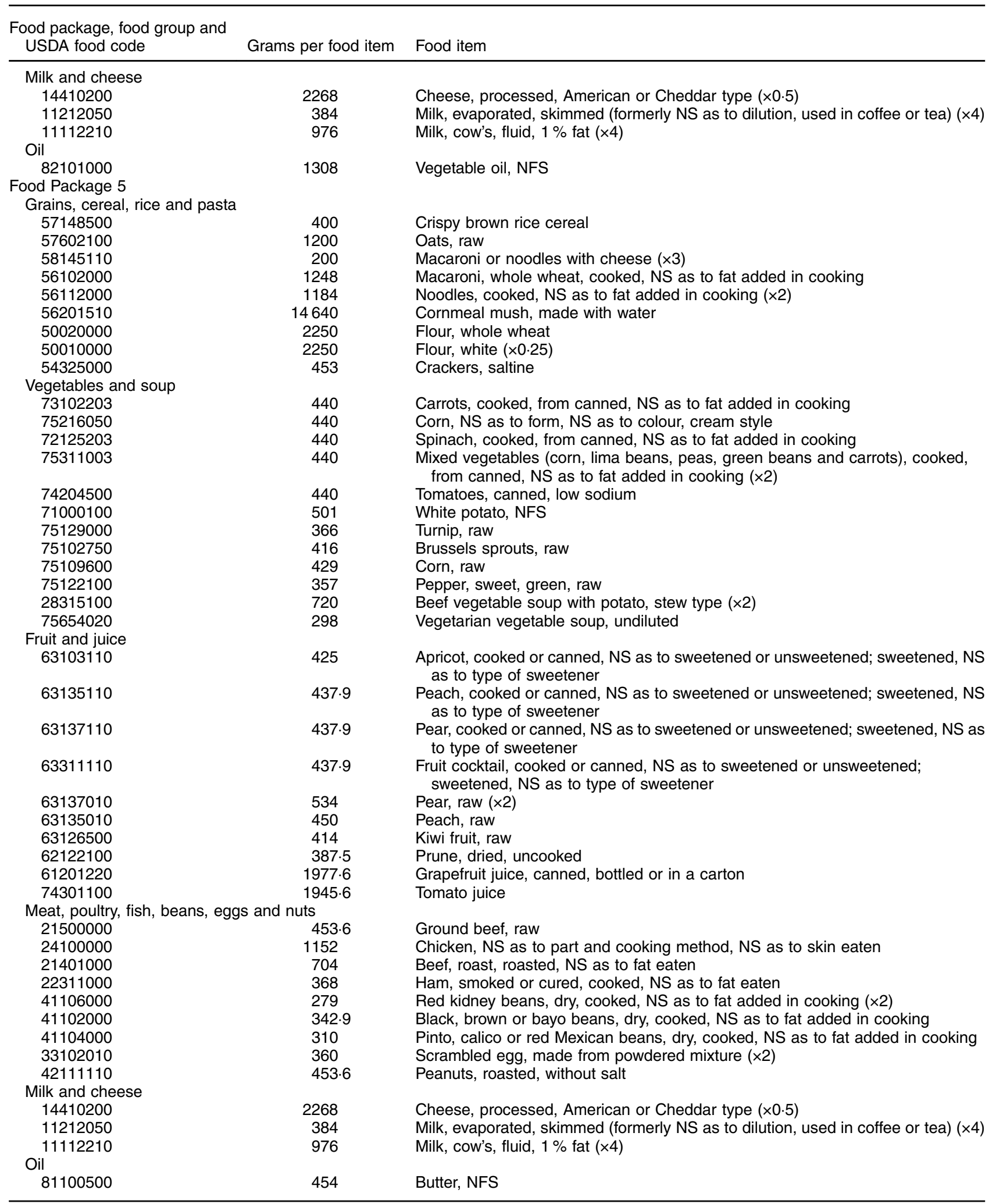

USDA, US Department of Agriculture; FDPIR, Food Distribution Program on Indian Reservations; NFS, not further specified; NS, not specified. 
Table $2 \mathrm{HEl}-2010 \dagger$ component and total scores for each of the five sample FDPIR monthly food packages $(n 5)$

\begin{tabular}{|c|c|c|c|c|c|c|c|c|}
\hline Component & $\begin{array}{l}\text { Maximum } \\
\text { value }\end{array}$ & $\begin{array}{l}\text { Standard for } \\
\text { maximum score }\end{array}$ & $\begin{array}{l}\text { Standard for } \\
\text { minimum score of } \\
\text { zero }\end{array}$ & Mean & SD & Range & $\begin{array}{l}\% \text { Meeting } \\
\text { maximum } \\
\text { value } \neq\end{array}$ & $n$ \\
\hline Total Fruit§ & 5 & $\begin{array}{l}\geq 0.8 \text { cup equivalent per } \\
1000 \mathrm{kcal}\end{array}$ & No Fruit & $3.52^{*}$ & 0.73 & $2 \cdot 60-4 \cdot 40$ & 0 & 0 \\
\hline Whole Fruitll & 5 & $\begin{array}{l}\geq 0.4 \text { cup equivalent per } \\
1000 \text { kcal }\end{array}$ & No Whole Fruit & $4 \cdot 60$ & 0.52 & $3 \cdot 90-5 \cdot 00$ & $40 \cdot 0$ & 2 \\
\hline Total Vegetables & 5 & $\begin{array}{l}\geq 1.1 \text { cup equivalents per } \\
1000 \mathrm{kcal}\end{array}$ & No Vegetables & $2 \cdot 58^{\star \star \star}$ & $0 \cdot 15$ & $2 \cdot 40-2 \cdot 80$ & 0 & 0 \\
\hline Greens and Beans & 5 & $\begin{array}{l}\geq 0.2 \text { cup equivalent per } \\
1000 \mathrm{kcal}\end{array}$ & $\begin{array}{l}\text { No Dark Green } \\
\text { Vegetables or } \\
\text { Beans and Peas }\end{array}$ & $0.92^{\star \star \star}$ & 1.00 & $0.00-2.20$ & 0 & 0 \\
\hline Whole Grains & 10 & $\begin{array}{l}\geq 1.5 \mathrm{oz} \text { equivalents per } \\
1000 \mathrm{kcal}\end{array}$ & No Whole Grains & $7 \cdot 88$ & 3.68 & $1 \cdot 50-10 \cdot 00$ & $60 \cdot 0$ & 3 \\
\hline Dairy†† & 10 & $\begin{array}{l}\geq 1 \cdot 3 \text { cup equivalents per } \\
1000 \mathrm{kcal}\end{array}$ & No Dairy & $5 \cdot 12^{\star \star \star}$ & 0.63 & $4 \cdot 20-5 \cdot 70$ & 0 & 0 \\
\hline 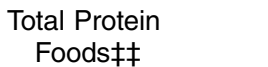 & 5 & $\begin{array}{l}\geq 2.5 \mathrm{oz} \text { equivalents per } \\
1000 \mathrm{kcal}\end{array}$ & No Protein Foods & $4 \cdot 14^{\star}$ & 0.56 & $3 \cdot 30-4 \cdot 80$ & 0 & 0 \\
\hline $\begin{array}{l}\text { Seafood and Plant } \\
\text { Proteins } \ddagger \ddagger, \S \S\end{array}$ & 5 & $\begin{array}{l}\geq 0.8 \mathrm{oz} \text { equivalent per } \\
1000 \mathrm{kcal}\end{array}$ & $\begin{array}{l}\text { No Seafood or } \\
\text { Plant Proteins }\end{array}$ & $4 \cdot 64$ & 0.53 & $3 \cdot 80-5 \cdot 00$ & $60 \cdot 0$ & 3 \\
\hline Fatty Acids IIII & 10 & $(P U F A+M U F A) / S F A>2.5$ & $\begin{array}{l}(\text { PUFA }+ \text { MUFA }) / \\
\text { SFA } \leq 1.2\end{array}$ & $4 \cdot 80$ & 4.55 & $0.00-10 \cdot 00$ & $20 \cdot 0$ & 1 \\
\hline Refined Grains & 10 & $\begin{array}{l}\leq 1.8 \text { oz equivalents per } \\
1000 \text { kcal }\end{array}$ & $\begin{array}{l}\geq 4.3 \mathrm{oz} \\
\quad \text { equivalents per } \\
1000 \text { kcal }\end{array}$ & $3.04^{\star *}$ & $2 \cdot 90$ & $0.00-6.40$ & 0 & 0 \\
\hline Sodium & 10 & $\leq 1.1 \mathrm{~g}$ per $1000 \mathrm{kcal}$ & $\begin{array}{l}\geq 2.0 \mathrm{~g} \mathrm{per} \\
1000 \mathrm{kcal}\end{array}$ & $5 \cdot 08^{\star}$ & $3 \cdot 15$ & $0 \cdot 70-9.30$ & 0 & 0 \\
\hline $\begin{array}{l}\text { Empty Caloriesףी } \\
\text { Total }\end{array}$ & $\begin{array}{r}20 \\
100\end{array}$ & $\leq 19 \%$ of energy & $\geq 50 \%$ of energy & $\begin{array}{l}20 \cdot 00 \\
66 \cdot 38^{\star \star}\end{array}$ & $\begin{array}{c}0 \\
11 \cdot 60\end{array}$ & $\begin{array}{l}20 \cdot 00-20 \cdot 00 \\
49 \cdot 50-79.50\end{array}$ & $100 \cdot 0$ & 5 \\
\hline
\end{tabular}

HEI-2010, Healthy Eating Index-2010; FDPIR, Food Distribution Program on Indian Reservations.

$1000 \mathrm{kcal}=4184 \mathrm{~kJ}$.

${ }^{\star} P<0.05 ;{ }^{* *} P<0.01 ;{ }^{* *} P<0.001$.

†Intakes between the minimum and maximum standards are scored proportionately.

łlncludes the five sample monthly food packages.

§Includes fruit juice.

IIIncludes all forms except juice.

IIncludes any beans and peas not counted as Total Protein Foods.

t†lncludes all milk products, such as fluid milk, yoghurt and cheese, and fortified soya beverages.

$\ddagger \ddagger$ Beans and peas are included here (and not with vegetables) when the Total Protein Foods standard is otherwise not met.

$\S \S$ Includes seafood, nuts, seeds, soya products (other than beverages) as well as beans and peas counted as Total Protein Foods.

IIIIRatio of PUFA and MUFA to SFA.

१ๆCalories from solid fats, alcohol and added sugars; threshold for counting alcohol is $>13 \mathrm{~g} / 1000 \mathrm{kcal}$.

found for Total Fruit, Total Vegetables, Greens and Beans, Dairy, Refined Grains, Total Protein Foods and Protein ${ }^{(36)}$. The current study shows that, although there was no significant difference, the HEI-2010 scores for Whole Fruit, Whole Grains, Seafood and Plant Proteins and Fatty Acids also fell short of the maximum HEI-2010 score, indicating a potential need to improve options within these categories. Although the HEI-2010 mean total score for FDPIR (score of 66) was slightly better than the American food supply (HEI-2005 score of 55) ${ }^{(44)}$, the FDPIR should target providing more inadequately consumed foods (of fruits, vegetables, whole grains or dairy) to promote better nutrition among participants in line with the needs of the American population.

Interestingly, HEI-2010 scores of each of the five assessed food packages show significant variation in nutritional quality and thereby emphasize the role of FDPIR centres in providing more foods that are consistent with adequacy components and fewer foods categorized as moderation components by HEI- $2010^{(38)}$. Secondly, consumer behaviour in making dietary choices from available food access should also be considered. Findings from the present study highlight opportunities to provide guidance to FDPIR participants about nutritionally balanced food choices at FDPIR centres as well as foods that participants acquire outside the FDPIR. The FDPIR should ideally provide participants with the opportunity to increase diet quality beyond the average American diet as well as meet the current DGA ${ }^{(36)}$. The FDPIR is positioned to modify its food and education offerings to best support lifestyles towards prevention of diet-related chronic disease.

The HEI-2010 FDPIR score from the current research (score of 66) resulted to be lower than a previous assessment of FDPIR that utilized HEI-2005 (score of $87)^{(34)}$. Although methodologies between FDPIR assessments differed, it is important to explore the differences found using the two versions of the Healthy Eating Index, which reflects the most up-to-date dietary guidance. The current assessment offered similar scores for Total Fruit, Whole Fruit, Total Vegetables, Greens and Beans (previously Dark Green and Orange Vegetables and Legumes), Dairy (previously Milk) and Empty Calories compared with the previous assessment ${ }^{(34)}$. Differences in 
scores between the two assessments can be attributed partially to foods selected in the food packages and partially to updates in scoring. For example, in the previous assessment that used HEI-2005, the component of Total Grains received a maximum score of 5 , while the current assessment for Whole Grains resulted in a score lower than the maximum ( 8 out of 10) and Refined Grains resulted in score significantly lower than the maximum (3 out of 10$)^{(34)}$. Grain foods randomly selected for the current assessment were split into the updated categories of Refined Grains and Whole Grains. Grain foods in the previous assessment were placed in the Total Grains category. In one additional example, the component of Oils and Saturated Fats scored relatively close to the maximum in the HEI-2005 analysis (9.8 out of 10), while in the current assessment Fatty Acids scored relatively low $(4.8 \text { out of } 10)^{(34)}$. This is in part due to the replacement of the Oils and Saturated Fats component with Fatty Acids in the HEI-2010. Improvements in the Refined Grain, Whole Grain and Fatty Acids categories are warranted. Although changes in national dietary guidance are usually minimal, these examples demonstrate the importance of assessing nutrition quality of FDPIR foods using new iterations of the Healthy Eating Index to capture important nuances in diet quality.

The HEI-2010 mean total score for FDPIR cannot be compared with other HEI-2010 scores in different food assistance contexts, as these analyses do not currently exist. Although there are limitations to comparing HEI-2005 and HEI-2010, the nutrient quality of the current FDPIR food packages using the HEI-2010 analysis is higher than of some other federal food assistance and nutrition programmes, including comparison to dietary intake of SNAP ${ }^{(34)}$ and Special Supplemental Nutrition Program for Women, Infants, and Children (WIC) $)^{(45)}$ participants using HEI-2005. Comparison of the FDPIR nutrient quality with SNAP and non-SNAP participants' dietary intake shows that the FDPIR has higher scores. These findings may be in part due to the analysis of actual participant dietary intake in SNAP and WIC, whereas the analysis in the current study measured nutrient quality of randomized food packages. Researchers working with the FDPIR should analyse dietary intake of FDPIR participants to understand the value of what nutrients are consumed in addition to the nutrient value of food package offerings. Specifically, the dietary intake of SNAP participants was found to have a HEI-2005 total score of 47 and nonparticipants were found to have a total score $51^{(46)}$, which is considerably lower than the average HEI-2010 mean score of 66 found in the present study for the nutrient quality of FDPIR packages. Furthermore, dietary intake of child participants in the WIC received a HEI-2005 total score of 58 , compared with dietary intake of children not participating in WIC who received a score of $60^{(45)}$, which are both lower than the mean nutrient quality score for the FDPIR food packages. The differences in findings may also be due to greater access to processed and sugar-added foods of SNAP compared with the FDPIR and lack of dietary analysis of intake of FDPIR participants. In contrast to SNAP where benefits can be used by participants to purchase 'foods of minimal nutritional value' including soda, water ices, chewing gum and candy, foods in the FDPIR package are selected to address some nutritional need $^{(47)}$. Participants in the FDPIR may also supplement their diet with purchased processed and sugar-added foods or other foods (e.g. hunted, grown, gathered), but the current analysis does not account for dietary intake.

The FDPIR still has nutritional shortcomings that need to be addressed in order to decrease the risk of diet-related chronic disease on American Indian reservations. In our current study and other observational work in progress, shortcomings of the FDPIR may derive from limited offerings of greens and total vegetables, nutrient profile of foods, sensory appeal of individual FDPIR offerings and the physical environment of the FDPIR centre, time needed to prepare FDPIR foods $v$. convenience foods, and lack of knowledge in preparing FDPIR foods. These issues are germane to improving diet quality of programme participants.

Increasing offerings of vegetables may require an increase in the budget allocated to the FDPIR if other aspects of the programme are to remain unchanged, given the relatively high price of produce in the USA compared with non-specialty crops. Modifying the structure of the FDPIR to offer greater selection of fresh fruits and vegetables may encourage produce consumption, particularly if this offering was coupled with nutrition information and cooking demonstrations on preparing recipes that are culturally compatible. In recent years, the quality of FDPIR food has been improved by the Fresh Fruits and Vegetables Program in which most individual FDPIR programmes now participate ${ }^{(47)}$. It will be important that these fresh fruit and vegetable offerings be kept fresh, or that canned or frozen produce is utilized, in order to retain maximum phytonutrients to benefit human health.

Given the variable HEI-2010 scores of different FDPIR food packages, directing food options to increase nutrient diversity would likely result in improved nutrition and health outcomes of participants. Healthy food choices may be encouraged through enhancing the sensory appeal of individual FDPIR offerings; for example, researchers should consider studying the consumer appeal components that FDPIR foods, packages and programme centres provide, as to the authors' knowledge no study has been conducted about the attractiveness of these variables to native populations. Additionally, increasing availability and diversity of culturally appropriate foods in specific food components that do not meet minimum recommendations would also assist in increasing the HEI-2010 score, specifically for Total Fruit, Total Vegetables, Greens and Beans, Dairy, Total Protein Foods, Refined Grains or Sodium. The addition of limes would add to overall 
availability of Total Fruit; replacing refined grains with whole grains such as wild rice, barley, quinoa, blue cornmeal, sorghum and rye has the potential to improve the Refined Grains score; and adding bison to the offerings would improve access to Total Protein Foods. Recently, Congress directed that a portion of FDPIR funding be used to purchase bison meat because of its low fat content and cultural value for American Indians, even if this is not tribally specific ${ }^{(47)}$.

There is promising opportunity to implement nutrition education and cooking demonstrations on how to supplement FDPIR offerings with culturally appropriate, accessible and healthy foods, especially since federal grant mechanisms exist to support nutrition education related to the FDPIR through the USDA Food Distribution Program Nutrition Education (FDPNE) ${ }^{(48)}$. Several successful initiatives have been launched to date that serve to enhance the food choices of FDPIR participants in culturally appropriate ways, including cooking demonstrations, taste tests, cooking competitions, gardening demonstrations with traditional foods, health wellness programmes and special events such as health fairs ${ }^{(47)}$.

The present study has several limitations that are important to address when interpreting findings and examining implications. As with many other studies that utilize the Health Eating Index to study nutritional adequacy in various settings (e.g. foods offered to children at child-care centres, foods offered to children through backpack programmes, the dollar menu displayed at a fast-food restaurant $)^{(43,44,49)}$, it is important to note that this evaluation of FDPIR involves analysis of food products and not actual consumer consumption. For example, the study assessed the quality of five randomly generated food packages of the FDPIR rather than actual food package selections made by participants. In addition, the study does not take into consideration other foods with which participants may supplement their food assistance packages such as local wild and cultivated foods or purchased foods. Finally, the availability of individual products is subject to market conditions, ITO and state agency orders, and seasonal availability. The current study did not limit USDA foods or options according to these factors and no published list is available to reflect that information to the authors' knowledge. Despite these limitations, the present study contributes to the sparse published literature assessing nutritional quality of a national food commodity programme geared toward a specific racial demographic.

There is a need for future studies to establish the linkages between FDPIR participation and long-term nutrition and health outcomes. Specifically, such future studies should examine the complex interplay between the FDPIR and other aspects of the food environment and food access, along with consumer lifestyle behaviour and dietary choices, food quality, genetics, epigenetics and food sovereignty. Studies that examine the HEI-2010 on actual FDPIR packages and diets of participants would further enhance the understanding of the contribution of this federal assistance programme to nutrition and health outcomes. Research on the FDPIR is particularly pressing because of the lack of available studies on the federal nutrition programme that serves an extremely vulnerable population in the USA that is at high risk of diet-related chronic disease ${ }^{(31)}$.

\section{Acknowledgements}

Acknowledgements: This research was requested by the Board of Directors of the National Association of Food Distribution Program on Indian Reservations. Financial support: This research was supported by the Montana State University College of Education Health and Human Development (C.B.S., S.A., H.H., T.S., Seed Grant); and Montana INBRE (IDeA Networks of Biomedical Research Excellence) (S.A., C.B.S., grant number 415-1081). Montana State University College of Education Health and Human Development and Montana INBRE had no role in the design, analysis or writing of this article. Conflict of interest: None. Authorship: All authors contributed to the concept and design, acquiring data, interpretation of data and drafting and revision of the manuscript. C.B.S., S.A. and H.H. obtained funding for this work. T.S. primarily conducted statistical analysis. C.B.S. directed manuscript development. Ethics of human subject participation: This research did not involve human subjects.

\section{References}

1. US Department of Health and Human Services (2010) The Surgeon General's Vision for a Healthy and Fit Nation. Rockville, MD: US Department of Health and Human Services, Office of the Surgeon General.

2. White M (2007) Food access and obesity. Obes Rev 8, Suppl. $1,99-107$.

3. Glanz K, Sallis JF, Saelens BE et al. (2005) Healthy nutrition environments: concepts and measures. Am J Health Promot 19, 330-333.

4. Befort CA, Nazir N \& Perri MG (2012) Prevalence of obesity among adults from rural and urban areas of the United States: findings from NHANES (2005-2008). J Rural Health 28, 392-397.

5. Sharkey JR (2009) Measuring potential access to food stores and food-service places in rural areas in the US. Am J Prev Med 36, 4 Suppl., S151-S155.

6. Powell LM, Slater S, Mirtcheva D et al. (2007) Food store availability and neighborhood characteristics in the United States. Prev Med 44, 189-195.

7. Beaulac J, Kristjansson E \& Cummins S (2009) A systematic review of food deserts, 1966-2007. Prev Chronic Dis 6, A105.

8. Larson NI, Story MT \& Nelson MC (2009) Neighborhood environments: disparities in access to healthy foods in the US. Am J Prev Med 36, 74-81.

9. Bower KM, Thorpe RJ Jr, Rohde C et al. (2014) The intersection of neighborhood racial segregation, poverty, and urbanicity and its impact on food store availability in the United States. Prev Med 58, 33-39.

10. Kaufman P, Dicken C \& Williams R (2014) Measuring Access to Healthful, Affordable Food in American Indian and 
Alaska Native Tribal Areas. Economic Information Bulletin no. EIB-131. Washington, DC: US Department of Agriculture, Economic Research Service.

11. Schiller J, Lucas J \& Peregoy J (2012) Summary Health Statistics for US Adults: National Health Interview Survey, 2011. Vital and Health Statistics Series 10 no. 256. Hyattsville, MD: National Center for Health Statistics.

12. Must A, Spadano J, Coakley EH et al. (1999) The disease burden associated with overweight and obesity. JAMA $\mathbf{2 8 2}$ 1523-1529.

13. American Diabetes Association (n.d.) American Indian/ Alaska Native Programs. http://www.diabetes.org/in-mycommunity/awareness-programs/american-indian-programs/ (accessed June 2014).

14. Basiotis PP, Lino M \& Anand R (1999) The Diet Quality of American Indians: Evidence from the Continuing Survey of Food Intakes by Individuals. Washington, DC: US Department of Agriculture, Center for Nutrition Policy and Promotion.

15. Huet C, Rosol R \& Egeland GM (2012) The prevalence of food insecurity is high and the diet quality poor in Inuit communities. J Nutr 142, 541-547.

16. Bauer KW, Widome R, Himes JH et al. (2012) High food insecurity and its correlates among families living on a rural American Indian Reservation. Am J Public Health 102, 1346-1352.

17. Mercille G, Receveur O \& Potvin L (2012) Household food insecurity and Canadian aboriginal women's self-efficacy in food preparation. Can J Diet Pract Res 73, 134-140.

18. Gordon A \& Oddo V (2012) Addressing Child Hunger and Obesity in Indian Country: Report to Congress. Princeton, NJ: Mathematica Policy Research.

19. Jernigan VBB, Salvatore AL, Styne DM et al. (2012) Addressing food insecurity in a Native American reservation using community-based participatory research. Health Educ Res 27, 645-655.

20. Edwards K \& Patchell B (2009) State of the science: a cultural view of native Americans and diabetes prevention. $J$ Cult Divers 16, 32-35.

21. Wiedman D (2012) Native American embodiment of the chronicities of modernity: reservation food, diabetes, and the metabolic syndrome among the Kiowa, Comanche, and Apache. Med Anthropol Q 26, 595-612.

22. Fleischhacker S, Byrd RR, Ramachandran G et al. (2012) Tools for healthy tribes: improving access to healthy foods in Indian country. Am J Prev Med 43, 3 Suppl. 2, S123-S129.

23. Kuhnlein HV \& Receveur O (1996) Dietary change and traditional food systems of indigenous peoples. Annu Rev Nutr 16, 417-442.

24. Compher C (2006) The nutrition transition in American Indians. J Transcult Nurs 17, 217-223.

25. Kappler CJ (1856) Treaty with the Blackfeet, 1855, pp. 736-740. Washington, DC: Government Printing Office.

26. Schamel CE, Rephlo M, Ross R et al. (1989) Guide to the Records of the United States House of Representatives at the National Archives, 1789-1989: Bicentennial Edition. Washington, DC: National Archives and Records Administration.

27. Luther Standing Bear (2006) Rations: a war-party: wild horses. In My People the Sioux, New Edition, pp. 71-81. Lincoln, NE: University of Nebraska Press.

28. Diedrich M (1991) There are a good many women and children that are naked and cannot come out of their tents (Little Hill, October 3, 1865). In Winnebago Oratory: Great Moments in the Recorded Speech of the Hochungra, 1742-1887, p. 27. Rochester, MN: Coyote Books.

29. United States Congress (1974) Commodity Distribution and Food Stamp Programs Hearings, Ninety-Third Congress,
Second Session, on S. 2871. Washington, DC: US Government Printing Office.

30. US Department of Agriculture, Food and Nutrition Service (2014) Food Distribution Program on Indian Reservations (FDPIR). http://www.fns.usda.gov/fdpir/food-distributionprogram-indian-reservations-fdpir (accessed Septemebr 2014).

31. Fox MK, Hamilton WL \& Lin B-H (2004) Effects of Food Assistance and Nutrition Programs on Nutrition and Health: Volume 4, Executive Summary of the Literature Review. Food Assistance and Nutrition Research Report no. FANRR-19-4. Washington, DC: US Department of Agriculture, Economic Research Service.

32. US Department of Agriculture, Food and Nutrition Service (2014) Food Distribution Program on Indian Reservations (FDPIR). About FDPIR. http://www.fns.usda.gov/ fdpir/about-fdpir (accessed June 2014).

33. US Department of Agriculture, Food and Nutrition Service (2014) Food Distribution Program Tables. http://www.fns. usda.gov/pd/food-distribution-program-tables (accessed June 2014).

34. Harper E, Orbeta R, Southworth L et al. (2008) FDPIR Food Package Nutritional Quality: Report to Congress. Special Nutrition Programs Report no. FD-08-FDPIR. Alexandria, VA: US Department of Agriculture, Food and Nutrition Service, Office of Research and Analysis.

35. US Department of Health and Human Services, National Institutes of Health, National Cancer Institute (2013) HEI Tools for Researchers. http://appliedresearch.cancer.gov/ tools/hei/tools.html (accessed November 2013).

36. Dietary Guidelines Advisory Committee (2010) Report of the Dietary Guidelines Advisory Committee on the Dietary Guidelines for Americans, 2010, to the Secretary of Agriculture and the Secretary of Health and Human Services. Washington, DC: US Government Printing Office.

37. US Department of Health and Human Services \& US Department of Agriculture (2005) Dietary Guidelines for Americans, 2005, 6th ed. Washington, DC: US Government Printing Office.

38. US Department of Health and Human Services, National Institutes of Health, National Cancer Institute (2014) Comparing the HEI-2005 \& HEI-2010. http://appliedresearch. cancer.gov/hei/comparing.html (accessed September 2014).

39. US Department of Agriculture, Food and Nutrition Service (2014) FNS Handbook 501 for FDPIR. http://www. fns.usda.gov/fdpir/fns-handbook-501-fdpir (accessed August 2014).

40. US Department of Health and Human Services, National Institutes of Health, National Cancer Institute (2013) Developing the Healthy Eating Index-2010. http://applied research.cancer.gov/tools/hei/developing.html (accessed November 2013).

41. Ahuja J, Montville J, Omolewa-Tomobi G et al. (2012) USDA Food and Nutrient Database for Dietary Studies, 5.0. Beltsville, MD: US Department of Agriculture, Agricultural Research Service, Food Surveys Research Group.

42. Center for Nutrition Policy and Promotion, US Department of Agriculture (n.d.) Healthy Eating Index Support Files 07 08. http://www.cnpp.usda.gov/healthy-eatingindex-support-files-07-08 (accessed April 2015).

43. Erinosho TO, Ball SC, Hanson PP et al. (2013) Assessing foods offered to children at child-care centers using the Healthy Eating Index-2005. J Acad Nutr Diet 113, 1084-1089.

44. Reedy J, Krebs-Smith SM \& Bosire C (2010) Evaluating the food environment: application of the Healthy Eating Index2005. Am J Prev Med 38, 465-471.

45. Cole N \& Fox MK (2008) Diet Quality of American Young Children by WIC Participation Status: Data from the National Health and Nutrition Examination Survey, 1999-2004. Special Nutrition Programs Report no. WIC-08-NH. Alexandria, 
VA: United States Department of Agriculture, Food and Nutrition Service, Office of Research, Nutrition and Analysis.

46. Gregory C, Ver Ploeg M, Andrews M et al. (2013) Supplemental Nutrition Assistance Program (SNAP) Participation Leads to Modest Changes in Diet Quality. Economic Research Report no. ERR-147. Washington, DC: US Department of Agriculture, Economic Research Service.

47. Finegold K, Pindus N, Levy D et al. (2009) Tribal Food Assistance: A Comparison of the Food Distribution Program on Indian Reservations (FDPIR) and the Supplemental
Nutrition Assistance Program (SNAP). Washington, DC: The Urban Institute.

48. US Department of Agriculture, Food and Nutrition Service (2014) Food Distribution Program on Indian Reservations (FDPIR). FDPIR Nutrition Education Grant Awards. http://www.fns.usda.gov/fdpir/fdpir-nutrition-education-grantawards (accessed September 2014).

49. Byker C \& Smith T (2015) Food assistance programs for children afford mixed dietary quality based on HEI-2010. Nutr Res 35, 35-40. 